\title{
Three-Dimensional Non-Destructive Inspection Using Novel Infrared-Terahertz Fusion Approaches ${ }^{+}$
}

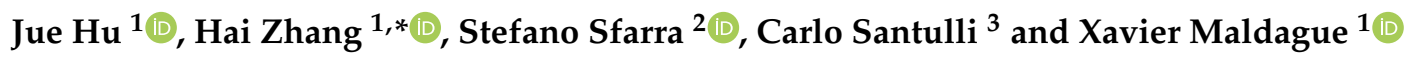 \\ 1 Computer Vision and Systems Laboratory, Department of Electrical and Computer Engineering, \\ Laval University, QC G1V 0A6, Canada; jue.hu.2@ulaval.ca (J.H.); xavier.maldague@gel.ulaval.ca (X.M.) \\ 2 Department of Industrial and Information Engineering and Economics (DIIIE), University of L'Aquila, \\ Piazzale E. Pontieri 1, Monteluco di Roio, 67100 L'Aquila, Italy; stefano.sfarra@univaq.it \\ 3 School of Architecture and Design, University of Camerino, 63100 Ascoli Piceno, Italy; \\ carlo.santulli@unicam.it \\ * Correspondence: hai.zhang.1@ulaval.ca \\ + Presented at the 16th International Workshop on Advanced Infrared Technology \& Applications, \\ 26-28 October 2021. Available online: https:/ / aita2021.sciforum.net/.
}

Citation: Hu, J.; Zhang, H.; Sfarra, S.; Santulli, C.; Maldague, X. ThreeDimensional Non-Destructive Inspection Using Novel InfraredTerahertz Fusion Approaches. Eng. Proc. 2021, 8, 24. https://doi.org/ 10.3390/engproc2021008024

Academic Editors: Giovanni Ferrarini, Paolo Bison and Gianluca Cadelano

Published: 24 November 2021

Publisher's Note: MDPI stays neutral with regard to jurisdictional claims in published maps and institutional affiliations.

Copyright: (c) 2021 by the authors. Licensee MDPI, Basel, Switzerland. This article is an open access article distributed under the terms and conditions of the Creative Commons Attribution (CC BY) license (https:// creativecommons.org/licenses/by/ $4.0 /)$.

\begin{abstract}
The imaging of structures with a complex material composition and geometry is still a challenge in the field of non-destructive testing (NDT). In this study, a non-invasive imaging technique is proposed for the non-destructive inspection of both cultural heritage and natural fiber composites. The proposed technique combines the surface information provided by infrared thermography (IRT) and the internal structure retrieved with terahertz (THz) time-domain spectroscopy using an unsupervised deep residual fusion network. Experiments show that the fusion results contain more material information than a single modality. In addition, 3D imaging has been achieved using the fusion results on natural fiber composites.
\end{abstract}

Keywords: infrared; terahertz; data fusion; cultural heritage; natural fiber composites

\section{Introduction}

Non-destructive testing (NDT) is a significant methodology both for composite material maintenance and cultural heritage conservation. Infrared thermography (IRT) is a well-known NDT technique, and it has the advantages of a fast inspection rate, the absence of contact, excellent spatial resolution and acquisition rate [1]. The heat transfer process through materials, which is the basis of IRT, determines its sensitivity for the detection of both surface and sub-surface defects. Terahertz time-domain spectroscopy (THz-TDS) is a non-invasive, high-resolution imaging technique which can discriminate between materials effectively [2]. However, complex material composition and geometry suppress the signal-to-noise ratio (SNR) of imaging on the internal defects using a single imaging modality.

To tackle this problem and provide high-contrast imaging results, a non-invasive imaging technique is proposed here for non-destructive inspection on both cultural heritage objects and natural fiber composites. The proposed technique combines the surface information provided by IRT and the internal structure retrieved with THz-TDS using an unsupervised deep residual fusion network. Experiments show that the fusion results provide more material information than a single modality. In addition, 3D imaging has been achieved using the fusion results on natural fiber composites.

\section{Specimens}

As a representative sample of cultural heritage, an old book cover was investigated in this study. Photographs of the book cover of a 19th century book is shown in Figure 1. The book, entitled The Little Illustrated Student, was published in Paris, France. 


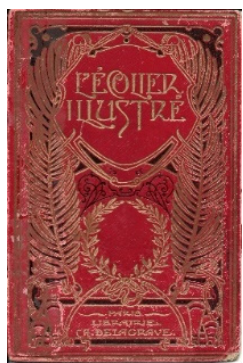

(a)

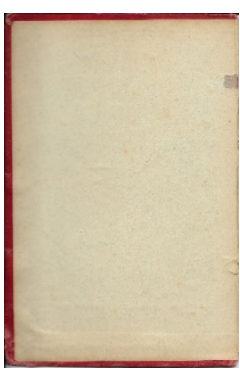

(b)

Figure 1. Book cover of a 19th century book: (a) Front side view; (b) Back side view.

Additionally, a natural fiber composite, mainly constituted by kenaf, was inspected using the proposed approach. The structure of the kenaf fiber composite is illustrated in Figure 2. In particular, the composite was realized with a hybrid method using both kenaf and glass fibers. A sandwich structure was realized at the end of the fabrication process. The core was fabricated with continuous kenaf/glass hybrid fiber yarns, whereas the two skins were made of a chopped strand matrix with a surface veil. The composite was later impacted in order to provoke damage partially visible to the naked eye.
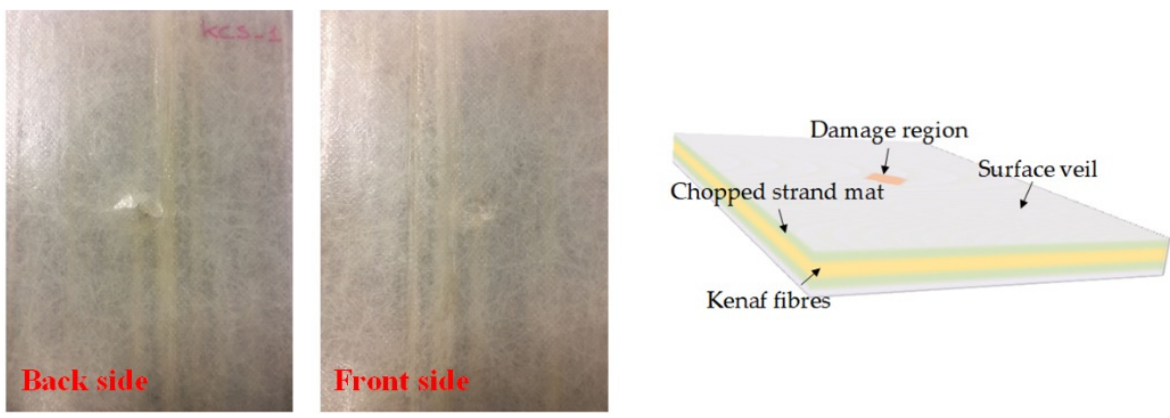

Figure 2. Photography and structure of the kenaf fiber composites.

\section{Infrared-Terahertz Fusion}

The proposed infrared-terahertz fusion approach is illustrated in Figure 3. Raw data collected from THz-TDS system was analyzed both in the time domain and frequency domain to obtain images based on different time domain representations and spectral characteristics of the materials.

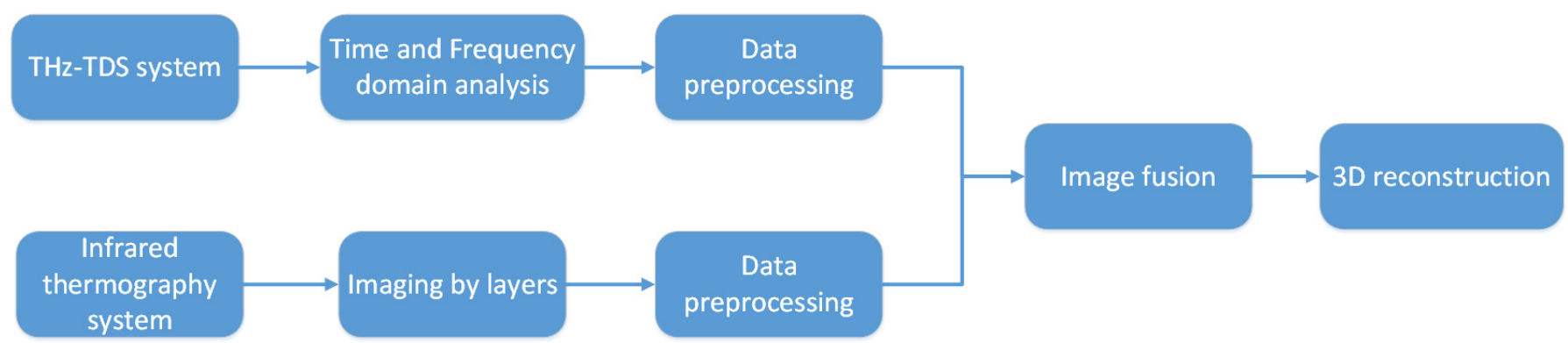

Figure 3. Schematic diagram of infrared-terahertz fusion approach.

The obtained thermal sequence from the IRT technique was also analyzed to image the different depths. Then, the analytical results from THz-TDS and IRT were pre-processed in order to confirm that the images from different techniques were in strict geometrical alignment and were intensity-matched. An unsupervised deep residual fusion network was developed to fuse the images from the different techniques (i.e., THz and IRT), and 
then the fused results were reconstructed in a 3D form to show the through-depth imaging results of the materials.

\section{Results}

Figure $4 \mathrm{c}$ shows the fused results on the book cover. The IRT imaging provided the texture details of it, along with the slight detachment, shown both at the upper and at the bottom right corner. However, the heat penetration depth limited the performance in inspecting internal defects. The $\mathrm{THz}$ imaging performed in transmission mode (Figure $4 \mathrm{~b}$ ) provided the internal defects and detachment clearly. The fusion imaging shown in Figure $4 \mathrm{c}$ combined both surface details (Figure 4a) and the internal defect information (Figure $4 \mathrm{~b}$ ). Moreover, the contrast of different materials was also improved.

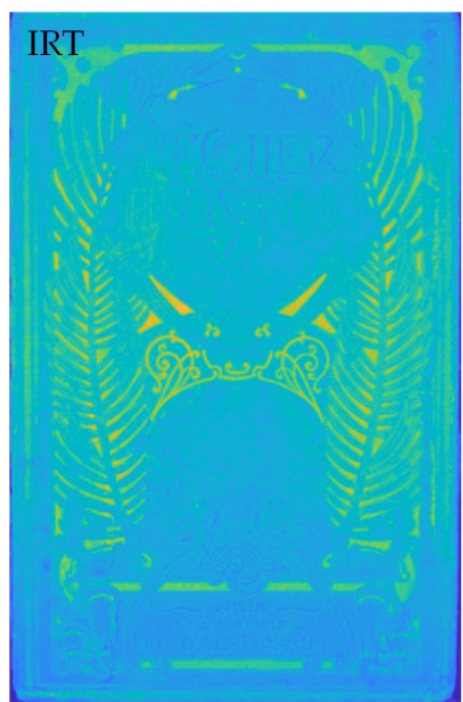

(a)

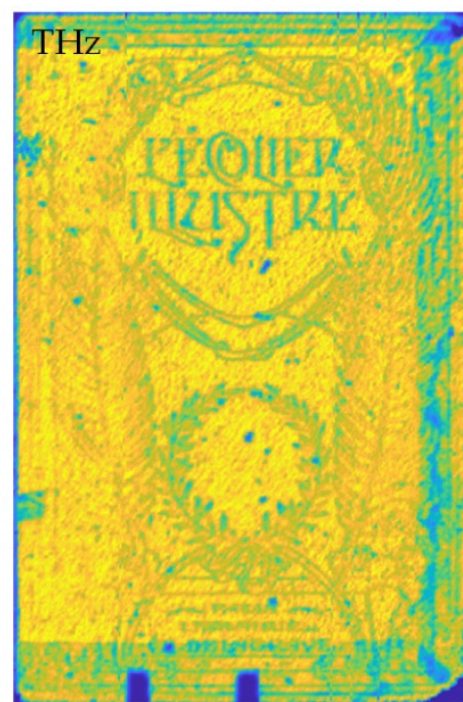

(b)

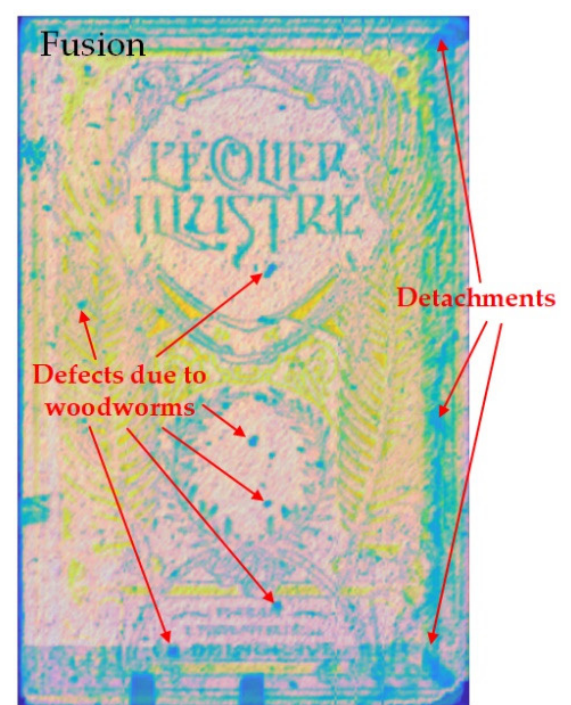

(c)

Figure 4. The fused results on the book cover: (a) IRT imaging; (b) THz imaging; (c) fusion imaging.

The 3D reconstruction results of the kenaf fiber composite are shown in Figure 5. In the second row of the figure, the images observed from different views of the 3D reconstruction model are reported. Figure $5 \mathrm{a}$ represents a cut on the chopped strand mat layer, compared with the X-ray computed tomography (CT) slice at the depth of $0.8 \mathrm{~mm}$ from the back panel. The low-velocity impact-induced damage and banded dents observed in IRT imaging are merged with the glass fiber content distribution viewed in $\mathrm{THz}$ imaging. All these patterns are presented in the fusion imaging results and can be mapped by the referred $\mathrm{X}$-ray CT slice. Figure $5 \mathrm{~b}$ represents a cut on the kenaf yarn layer, which refers to the CT slice obtained at the depth of $1.6 \mathrm{~mm}$ from the back panel. It is worth mentioning that, as an internal layer, the kenaf yarns are observed clearly with the impact-induced damage in the 3D fused result. Figure $5 \mathrm{c}$ shows the cross-section imaging cut at the position of 71.5 and $88.5 \mathrm{~mm}$ from the upper edge of the sample. It is interesting that the impact-induced damage appears at the position of $71.5 \mathrm{~mm}$ from upper edge. Therefore, the morphology of the impact can be observed in this cross-section imaging. Figure $5 \mathrm{~d}$ presents the horizontal section of the kenaf fiber yarn layer. As an internal layer in this unusual 3D structure, the kenaf yarn layer was chosen for the detailed comparison with the CT slice. The kenaf yarns present both on the cropped horizontal section of 3D fusion imaging and the cropped CT slice are matched by means of a red arrow. A total of 26 kenaf yarns can be mapped into the $\mathrm{X}$-ray $\mathrm{CT}$ inspection result, one-by-one. 


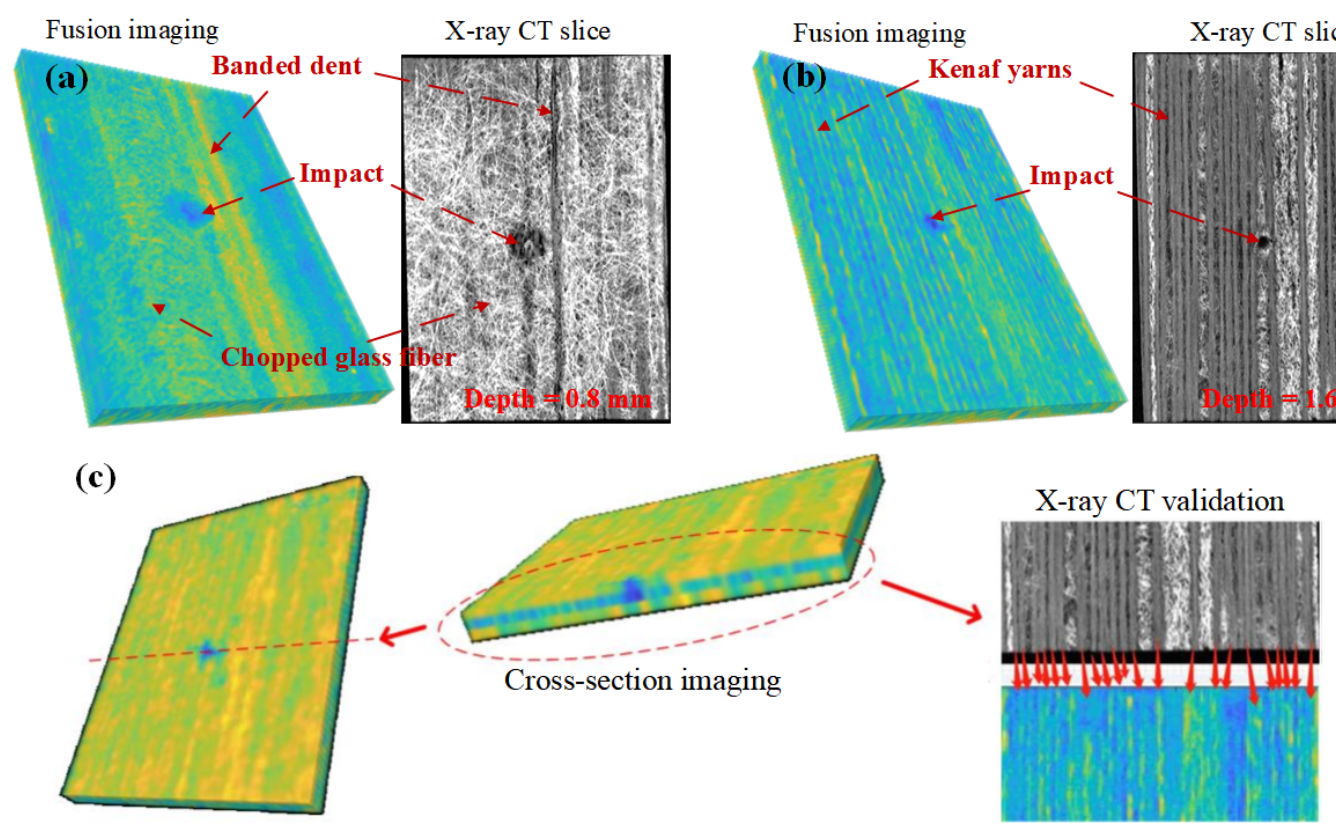

Figure 5. Three-dimensional fusion imaging results of the natural fiber composite. Images on the second row refer to the 3D model from different observation views. The top row $(\mathbf{a}, \mathbf{b})$ illustrates the comparison of the $3 \mathrm{D}$ reconstruction results cut from different depths and the X-ray CT imaging. The bottom row (c) shows the cross section and horizontal sections of the natural fiber composite.

\section{Conclusions}

In this study, a non-invasive imaging technique is proposed for non-destructive inspection of both cultural heritage objects and natural fiber composites. The proposed technique combines the surface information using IRT and the internal ones obtained via THz-TDS; an unsupervised deep residual fusion network is here developed for the first time. The results for an old book cover combine the defect information of two modalities and enhance the contrast in imaging. The results for a natural fiber composite using 3D imaging offer a better way to understand the characteristics of the composite itself. Comparison between the 3D fusion imaging results and X-ray $\mathrm{CT}$ inspection proves that the proposed fusion imaging technique has a promising performance in the imaging of different layers in such a complex composite structure.

Institutional Review Board Statement: Not applicable.

Informed Consent Statement: Not applicable.

Data Availability Statement: Data sharing not applicable.

Acknowledgments: This research was supported by the Canada Research Chair in Multipolar Infrared Vision (MiViM), the Natural Sciences and Engineering Research Council (NSERC) Canada through the Discovery Grant program and the Create-oN DuTy! program.

Conflicts of Interest: The authors declare no conflict of interest.

\section{References}

1. Zhang, H.; Sfarra, S.; Saluja, K.; Peeters, J.; Fleuret, J.; Duan, Y.; Fernandes, H.; Avdelidis, N.; Ibarra-Castanedo, C.; Maldague, X. Non-Destructive Investigation of Paintings on Canvas by Continuous Wave Terahertz Imaging and Flash Thermography. J. Nondestruct. Eval. 2017, 36, 34. [CrossRef]

2. Zhang, H.; Sfarra, S.; Osman, A.; Szielasko, K.; Stumm, C.; Genest, M.; Maldague, X.P. An Infrared-Induced Terahertz Imaging Modality for Foreign Object Detection in a Lightweight Honeycomb Composite Structure. IEEE Trans. Ind. Inform. 2018, 14, 5629-5636. [CrossRef] 